\title{
Book Scanner Dewarping with Weak 3d Measurements and a Simplified Surface Model
}

\author{
Erik Lilienblum and Bernd Michaelis \\ Institute for Electronics, Signal Processing and Communications \\ Otto-von-Guericke University Magdeburg \\ P.O.Box 4120, 39106 Magdeburg, Germany \\ \{erik.lilienblum, bernd.michaelis\}@ovgu.de
}

\begin{abstract}
For book scanner technologies projective distortions are the main problem. In general, the use of $3 \mathrm{~d}$ measurements of a warped surface is the best way to remove the projective distortions. But if the quality of the $3 \mathrm{~d}$ measurements is very low, it is difficult to get satisfying dewarping results. In our paper we present a new technique handling this problem by introducing a simplified surface model. We use this model as a basis to compute a linear approximation parallel to the geometrical position of the book crease. The resulting method leads to a robust and fast computation. It provides us with a reliable dewarping output even for weak measurements given by a light sectioning method of top view scanners.
\end{abstract}

\section{Introduction}

Digital archiving of hard-back literature increasingly becomes an essential part of the work of libraries and museums. Although for this purpose the modern computer technology already accomplishes major premises, the status quo of the scanner technology is not satisfying. In particular it is hardly possible to get distortion-free copies from thick books without damaging them. This currently represents a large problem both for high quality digitalisation of valuable historical books in museums and for simple copying of common books in public libraries. Besides, the image distortions bring a further problem with itself. A full automated character recognition (e.g. OCR) in the area of book crease is often impossible.

In the last years a special kind of book scanner, which is called top view scanner, became generally accepted on the market. To use the books in their natural way those scanners capture a copy of the page from above and from a certain distance. Through the use of a line camera with an appropriate stripe lighting it is possible to receive an evenly sharp and well illuminated two-dimensional image. However, we inevitably get a distorted copy in consequence of the projective geometry of the scanner and the warped surface of the page. An example can be seen on the upper left in figure 1

Removing the projective distortions supported by $3 \mathrm{~d}$ measurements of the page surface is a well known idea which we also use in our book scanner application. For the 3d surface measurement we extend the scanner construction by

D. Coeurjolly et al. (Eds.): DGCI 2008, LNCS 4992, pp. 529 540, 2008.

(C) Springer-Verlag Berlin Heidelberg 2008 


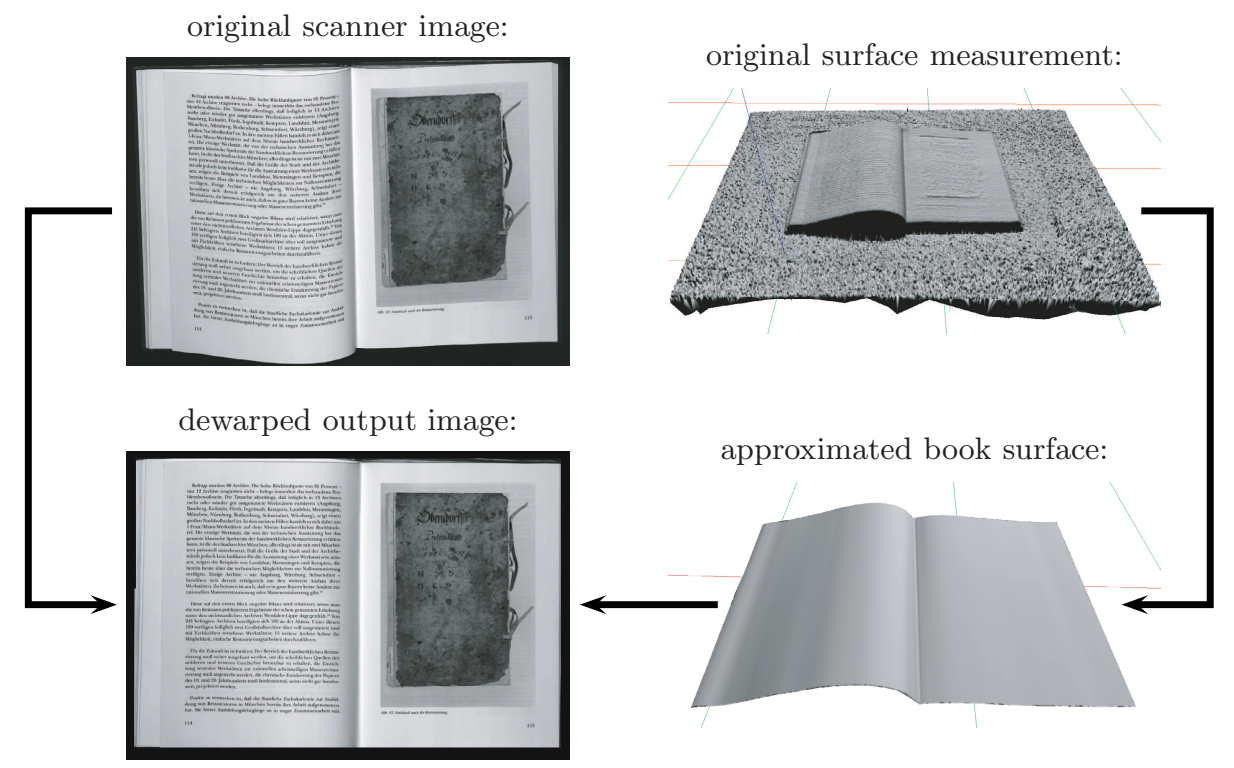

Fig. 1. Dewarping concept with 3d surface measurements

an additional matrix camera and apply a special kind of light sectioning. The result is represented on the upper right in figure 1. For homogeneous textures on the page this method provides sufficient accuracy. In contrast, if there are figures with very dark parts on the page, we get inapplicable measurements in these regions. Independent from the applied method this is a common problem for optical measurement systems.

However, to use the surface reconstruction despite their low quality in regions of weak measurements we have to approximate the book surface. The essence of our proposed method is to get a save and coherent surface reconstruction of the page, which is widely independent from the original measurement quality. The result of our approximation is represented down on the right in figure 1 . By combining our approximated surface reconstruction and the original scanner image we can calculate a nearly distortion-free copy of the page. An example of an output result is shown down on the left in figure 1 .

\section{Related Work}

Removing the projective distortion in a two-dimensional image of a three-dimensional warped surface is an abstract mathematical problem. A possibility without using additional information or hardware support offers the "shape from shading" principle. In [1/2 the shape of the surface is drawn directly from the distribution of brightness. Because of strict conditions the exactness and reliability of this approach are not particularly high. 
Another approach without an extension of the scanner hardware is to recognize the lines of text in a page 3415. Supposing that the lines must be straight, the original scanner image can be corrected accordingly. But this method works only with text pages. Pages with figures or insular text blocks can not be dewarped.

A quite similar technique is used in systems recognizing the margins. One approach is given by Rebmann et al. in [6]. Another approach and an example of its application can be found in a software tool which is based on the patent 7]. The patent describes a dewarping method disposing the geometrical position of the lower page margin. In most cases the software solution works well, but there are problems, if the book is not aligned well or if there are notes, which exceed the lower paper delimitation. These problems were a main motivation for us to develop an alternative solution.

Book scanner systems with additional hardware support are mostly based on the construction of a vision system and the calculation of a $3 d$ surface reconstruction of the page. There are constructions with one matrix camera and one projector in [8], two matrix cameras in [9] or laser triangulation in [10]. These techniques are often costly concerning the additional hardware and provide low resolutions in the copy of documents due to the exclusive use of matrix cameras. Additionally, there are a lot of other techniques to get a surface reconstruction, which we do not describe here. A good survey is given in [1].

The surface reconstruction of a book page is the basis to solve the dewarping problem. The success of an approach depends more on the quality of the $3 \mathrm{~d}$ data than on the quality of the dewarping algorithm. For exact measurements the approach from Brown 12 should provide a good dewarping result even for rigid surface deformations. But computational costs of that approach are very high and it is difficult to bear measurement errors. For this reason we prefer the works from Liang [1314. As in our own approach it integrates the mathematical model of a warped page.

\section{$3 \quad$ Weak Surface Measurements}

As described in [15] and patented in [16] our approach to calculate the surface reconstruction is based on a further development of classical light sectioning [17. According to this well known principle the position of the light stripe in an image of the matrix camera depends on the surface shape of the page we are scanning. Thereby from the captured image sequence we can infer the form of the whole surface. Figure 2 shows a schematic representation of our setup.

In classical light sectioning one determines in an image of the matrix camera the local pixel position of a projected light stripe. Usually this only works if the light stripe is very small, e.g. a projected laser line. Because the stripe lighting of a top view scanner generates a broad light stripe we have to apply an alternative technique. For a pixel of the matrix camera we determine from the image sequence the point in time, on which the light stripe was passing the pixel. Independent from the width of the stripe lighting we can calculate the point in 


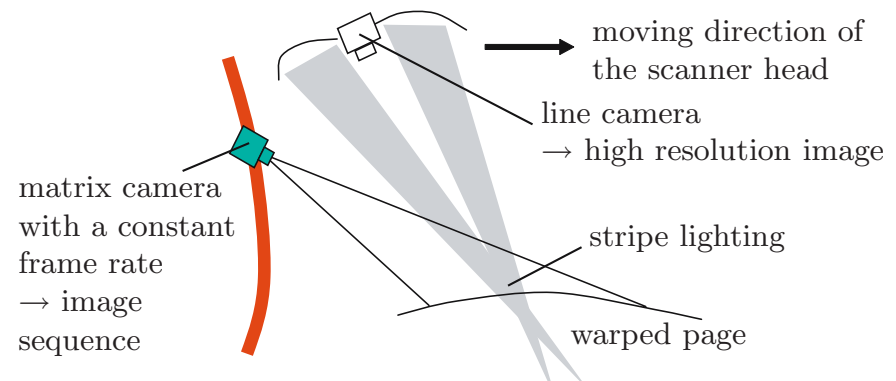

Fig. 2. Schematic representation of our setup

time with comparative high accuracy. By triangulation of the optic beam of the pixel and the plane of the stripe lighting we get the position of a surface point.

Evaluating the measurements of our method partially unveils some notable deviations between measured values and actual values. These measurement errors are not due to incorrectly computed time values or errors in the calibration of the system. They are caused by inhomogeneous paper properties and reflections. Especially at very dark textures the light of the surrounding areas has a large influence. That leads to weak measurements which are shown in figure 3 .
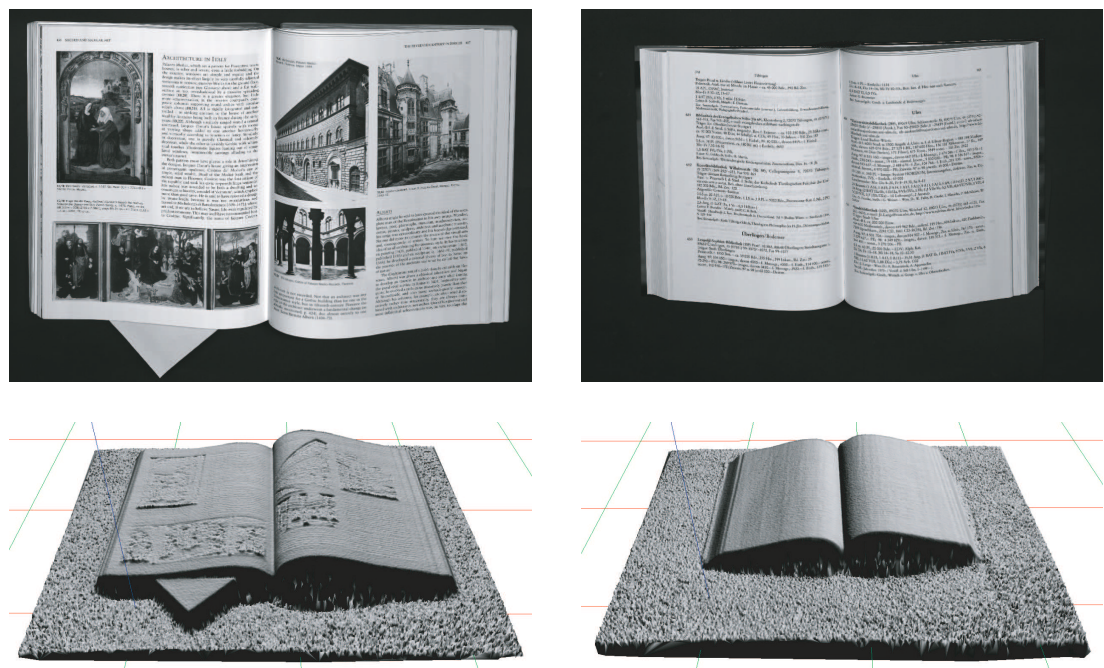

Fig. 3. Weak 3d measurements (left) and good $3 \mathrm{~d}$ measurements (right)

The rough $3 \mathrm{~d}$-surface of the left example in figure 3 is caused by errors of weak measurements. Especially at the dark parts in the illustration of the page we get notable measurement deviations, which we cannot level out by local 
operations. On the right side of figure 3 we see an example with a proper $3 \mathrm{~d}$ surface measurement.

Weak surface measurements are not only a problem of our time based light sectioning method. The quality of all approaches mentioned in section 2 depend on the page texture. Generally, we can state that the more we invest in the scanner hardware and the calculation time the fewer weak measurements we get. Both a better scanner hardware and a higher calculation time cause costs, which we can avoid by developing an efficient dewarping method working robust with weak measurements.

\section{The Book Model Calculation}

\subsection{Assumptions}

The approximation of a surface can be computed under very different points of view. If the quality of the given measurements is low, information about the surface characteristics like smoothness or boundary conditions are very important. Especially the kind of the approximation method should depend on the purpose of the expected approximation result.

In case of book surface approximation we know that the page surface is very smooth, normally. Therefore we can level out local measuring noise by simply choosing a large approximation area. But this procedure is not sufficient in case of weak or missing measurements, which are often locally connected. Here we need more stringent conditions, which we define for our special approximation problem through a geometric book model. The price we have to pay for introducing a stringent book model is the inability to dewarp rigid deformed books. But the advantage is that we can develop an algorithm which is fast, robust, and applicable for nearly all other kinds of books.

The definition of the book model is based on the following main assumptions:

1. There is a book crease which is detectable in the $3 \mathrm{~d}$ measurement set.

2. Parallel to the book crease there is only a very small surface warping.

Clearly, both assumptions do not claim completeness for all kinds of books. But this is rarely a problem. A book without a crease is very unusual and we can exclude such cases for our proposed method by an exception rule. The second point, the warping direction, is more difficult because for instance a dog-ear cannot be mapped by our model. But generally dog-ears should not possess a main meaning for book scanners.

\subsection{Improving the Crease Detection}

The most important characteristics of our book model is the geometric location of the book crease. It subdivides the book surface into two parts and gives the orientation of the pages. The easiest way to detect the book crease is searching a minimum line in the middle of the $3 \mathrm{~d}$ measurement set. 
In case of weak measurements this procedure provides us only with a first approximation. To improve this result we have to analyse the surface measurements in the neighbourhood of the book crease. Therefore we calculate a linear approximation of the surface through fitting a plane on the left and the right side of the crease, respectively. The planes which we denote by $E_{l}$ and $E_{r}$ are schematically shown in figure 4.

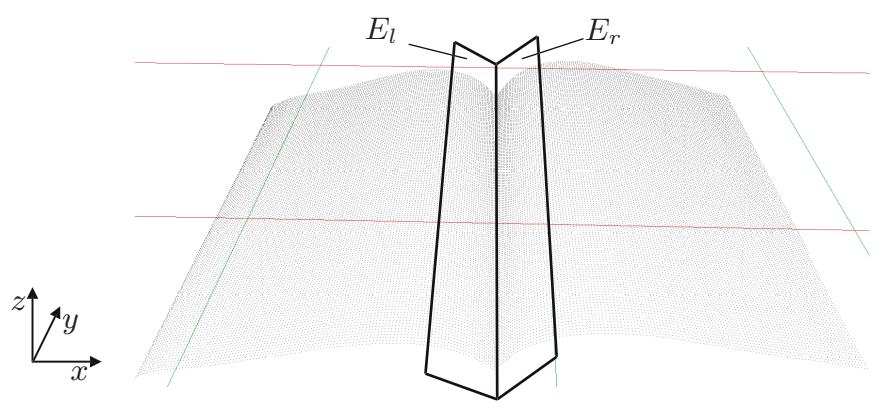

Fig. 4. Approximation planes left and right from the book crease

Let be given the first calculation of the geometric location of the book crease, which we get by searching the minimum line. Our improvement refers to the direction of the book crease in the telecentric view to the $x y$-level. In this case we can set $z=0$ and describe the geometric location of the crease in the $x y$-level with the normal form for straight lines

$$
a x+b y+c=0 .
$$

We now compute the approximation planes $E_{l}$ and $E_{r}$ given through

$$
\begin{gathered}
E_{l}: \quad A_{l} x+B_{l} y+C_{l} z+D_{l}=0, \\
E_{r}: \quad A_{r} x+B_{r} y+C_{r} z+D_{r}=0 .
\end{gathered}
$$

Then we form an intersection between the planes and project the result into the $x y$-level. Thus we get in the $x y$-level an improvement of equation 1 with

$$
\begin{aligned}
a & =A_{r}-A_{l}, \\
b & =B_{r}-B_{l} .
\end{aligned}
$$

The parameter $c$ of equation 1 cannot be improved by this method because the intersection of the approximation planes can induce a displacement. After correcting $a$ and $b$ parameter $c$ has to be recomputed by searching the minimum.

The exact alignment of the book crease has a direct effect on the alignment of the output result. An example of the consequence of a displaced book crease is given in figure 5. Here the difference between the uncorrected book crease and the corrected book crease is about 2 degrees. That sounds not much, but it is important for the visual overall impression of the dewarped output. With the described method we typically gain error corrections within 2 and -2 degrees. 


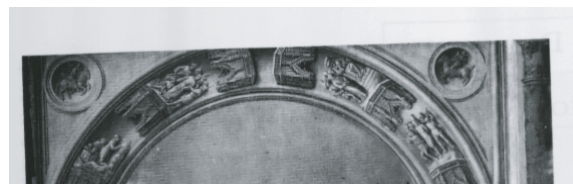

ARCHITECTURE IN ITALY

Palazzo Medici, which set a pattern for Florentine townhouses, is sober and severe, even a little forbidding. On the exterior, windows are simple and regular and the design makes its effect largely by very carefully adjusted

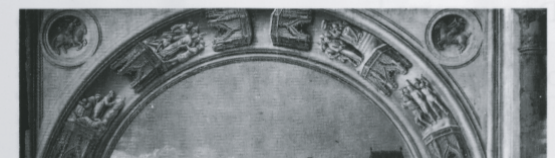

\section{ARCHITECTURE IN ITALY}

Palazzo Medici, which set a pattern for Florentine townhouses, is sober and severe, even a little forbidding. On the exterior, windows are simple and regular and the design makes its effect largely by very carefully adjusted

Fig. 5. Uncorrected and corrected alignment of the book crease

\subsection{Weighted Linear Approximation}

The second point of our book model definition gives us the possibility to level out the weak measurements by linear approximation patches over the complete vertical page length. Because this computation only leads to an error reduction similar to forming an average we have to introduce a quality estimation of the used measurements. Because in our light sectioning method dark surface areas are influenced by the surrounding light, the brightness of a measured point can be a good value for this purpose. This certainly is a compromise but it is also an important condition to develop a robust and fast method based on a linear approximation of weighted measurements.

The set of weighted measurements that goes into one linear approximation we define by

$$
\Gamma\left(d_{1}, d_{2}\right)=\left\{\left(p, \nu(p)^{2}\right) \mid p \in M, d_{1} \leq \kappa(p) \leq d_{2}\right\}
$$

whereby $\kappa(p)$ is the Euclidean distance between a point $p$ and the detected book crease relating to the $x y$-level, $d_{1}$ and $d_{2}$ are constraints to set the boundaries of the approximation area, $M \subset \mathbb{R}^{3}$ is the set of measured surface points, and $\nu(p)$ is the brightness of a measured point.

The actual operation to fit a plane into a set of weighted points is a standard problem. We do not describe it here. We denote that fitting operation with $P$, whereby the result of the operation $P: M \rightarrow \mathbb{R}^{3}$ is defined by an infinite set of $3 \mathrm{~d}$ points forming a plane. We now can form a straight line defined by the set

$$
S(i, g)=\{p \mid p \in P(\Gamma((i-1) r,(i+1) r)) \text { and } \kappa(p)=i r\},
$$

whereby $i \in \mathbb{G}$ is an integer value we call grid coordinate and $r \in \mathbb{R}$ is a real value we call grid distance. Through a computation of these straight lines for all $i$ we get an equidistant grid of approximation values along the whole book surface. An example can be seen schematically in figure 6.

The new format of our $3 \mathrm{~d}$ approximation values provides us with good properties for the further processing. We are easily able to detect the page borders by analysing the difference between the original measurements and the linear approximation. Such a 3d supported border recognition is much more stable than 


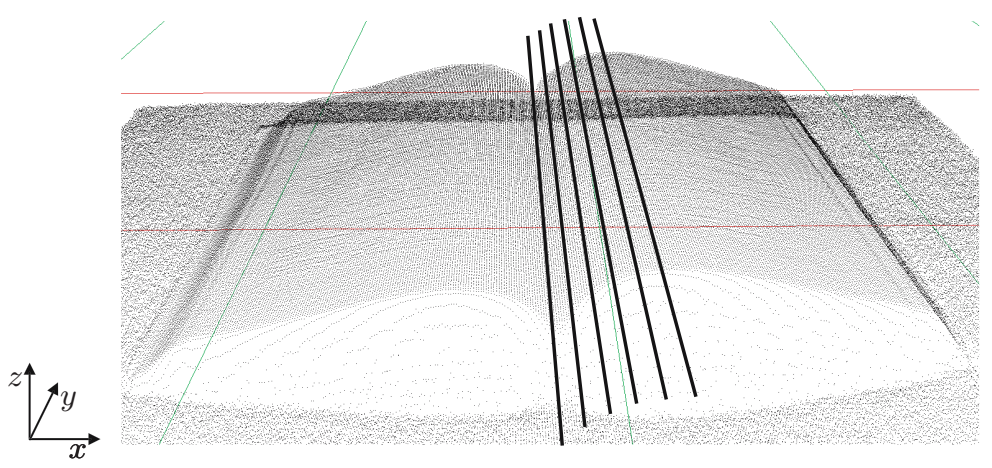

Fig. 6. Linear approximation with straight lines over the vertical book length

a brightness supported border recognition. The result is convincing even if there are notes going beyond the page border.

\subsection{Dewarping and Output}

After applying the weighted linear approximation of the page surface we have got an aligned equidistant measurement grid. To receive a dewarped output result we have to develop the warped surface into a plane. For this our data structure of straight lines is a good basis.

We start the dewarping algorithm from the book crease toward the outer delimitation of the left and right page, respectively. In each step we fold the $3 \mathrm{~d}$ distance between two neighbouring approximation lines into the $x y$-level. Thereby we get a new line in a $2 \mathrm{~d}$ output coordinate system. The height information of each line is saved in a relation to the original line.

In figure 7 a we see the origin in a telecentric view toward the $x y$-level of our $x y z$-coordinate system. It schematically diagrammes the detected book crease and the first 5 approximation lines to the right in its original geometrical direction. In figure [7. $\mathrm{b}$ we see the approximation lines in a transformed $x^{\prime} y^{\prime} z$ coordinate system aligned to the book crease. The $x^{\prime} y^{\prime}$-level of the transformed $x^{\prime} y^{\prime} z$ coordinate system is equal to the $2 \mathrm{~d}$ output $u v$-coordinate system.

In the $u v$ coordinate system we fold the approximation lines into the plane which is shown in figure 7 c. Through the transformation of the surface into a plane we loose the equidistant grid structure and the straight lines are not longer aligned. As it is shown in figure 7]d we renew the grid structure through the computation of equidistant supporting points by a linear interpolation. Thereby we save the relation to the original $3 \mathrm{~d}$ points. As a result we obtain at the $u v$ coordinate system an equidistant set of supporting points with a relation to $3 \mathrm{~d}$ points of the approximated book surface. This data structure is a direct basis to generate a dewarped output image of the page.

The calibration of the complete book scanner system provides us with a mapping from $3 \mathrm{~d}$ points into the original scanner image. From this mapping we 

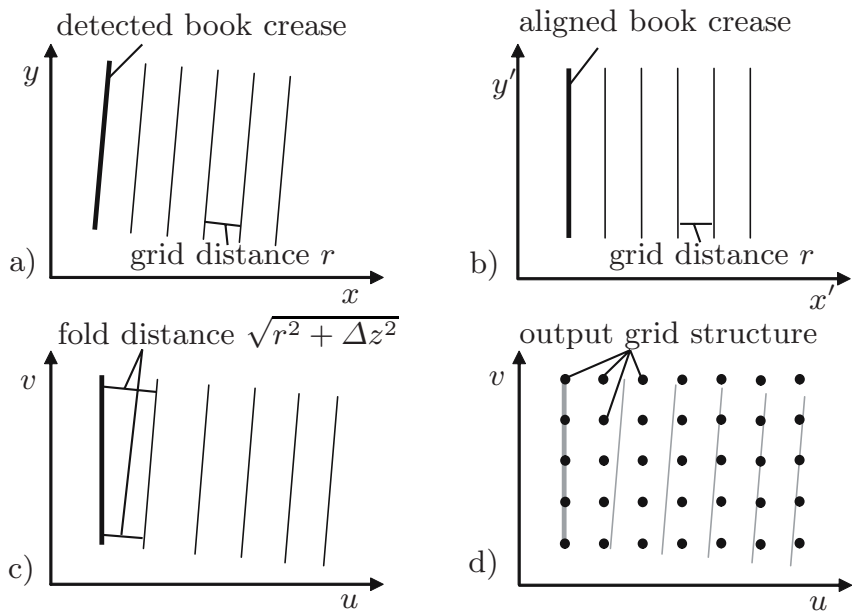

Fig. 7. Dewarping steps after the linear approximation

obtain for any point in the $3 \mathrm{~d}$ space a subpixel coordinate in the scanner image with high accuracy. To generate a dewarped image we only have to output the subpixel values, which we get by mapping the supporting points. We get a defined high resolution equal to the original scanner image by bilinear interpolation between the supporting points related to our equidistant grid.

\section{Results}

It is difficult to evaluate the results of our method by objective mathematical calculations. On one hand the evaluation depends much on the quality and characteristics of the input book. On the other hand there is no standard evaluation method to other dewarping algorithms. So we present here some examples with different book characteristics, which we analyse concerning the dewarping quality of the output.

At first we show in figure 8 the dewarped output of the two examples from figure 3 in section 3 It gives a general impression of the ability of our dewarping method. A more detailed illustration is already shown on the left in figure 5 The text lines are nearly straight and well aligned. There are no problems with the illustrations even if they include deep black parts. Additionally, the characters situated near the book crease appear in there original size. We still get sufficient results even if the book crease is very deep like in the example of figure 9 .

The examples are only a small selection of our test scans with many different books. If near the book crease the brightness is high enough we detect the correct geometrical location with nearly $100 \%$. The detection only fails if there is no book crease at all or the geometric form of the book crease is very flat. In this case we cannot apply the method presented in this paper. 

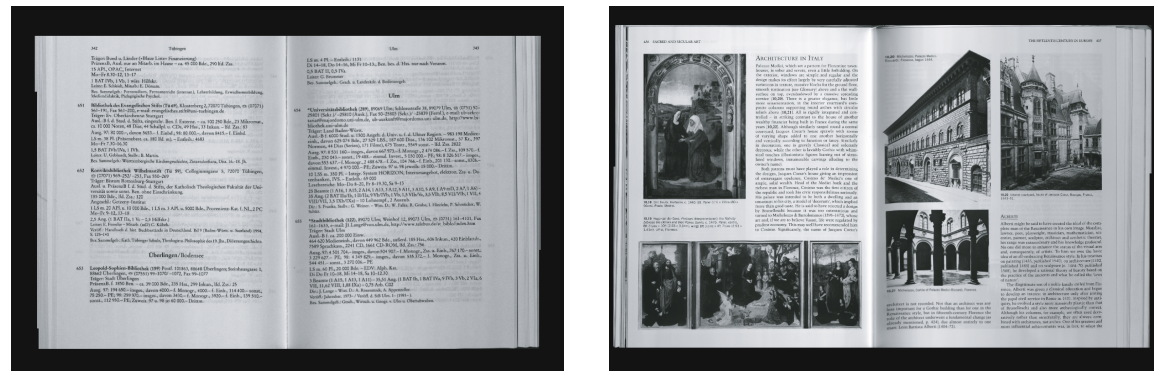

Fig. 8. Dewarped output results
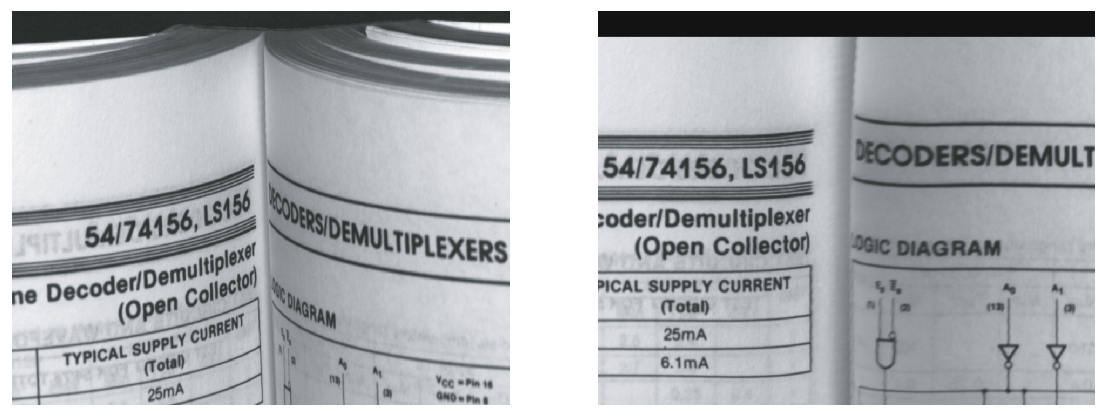

Fig. 9. Character dewarping near a deep book crease

If we detect a book crease the method works robust and fast. On a standard $\mathrm{PC}$ with $3.4 \mathrm{GHz}$ the computation time for our method is below 1s. Within this period we have to calculate up to $640 \times 4483 \mathrm{~d}$ points according to the resolution of the matrix camera. Without dewarping the pure scanning time of our book scanner with a resolution of $300 \mathrm{dpi}$ is about $1 \mathrm{~s}$. That means we receive a total time of 2 s for generating a nearly distortion-free book copy. This is a very good value for a publicly used book scanner system.

The robustness of the method depends on the distribution of the brightness values along the book surface. In most cases we get the expected completely dewarped output results. Only if there are deep black values over the whole vertical length of the page our method yields incorrect output results, e.g. a wrong outer delimitation of the page or additional distortions not coming from the original scanner image. An examples is given in figure 10. While on the left page the dewarping is nearly correct, we see on the right page deviations from the expected output, which are due to weak measurements over the vertical length of the page. We can stop such incorrect outputs through defining some consistency rules relating the brightness values, but we cannot sufficiently correct them. The analysis and improvement of this infrequent problem will be one of the next steps our research work. 

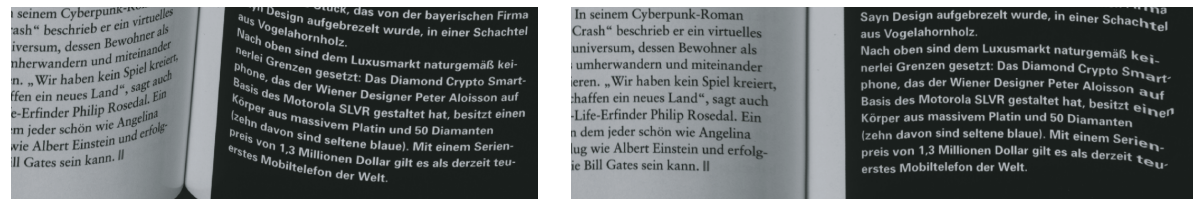

Fig. 10. Example of incorrect output due to low vertical brightness values

\section{Conclusion and Further Work}

We have presented an improved method to generate nearly distortion-free copies of bounded books. As input we use the original images of a top view scanner and a set of $3 \mathrm{~d}$ surface measurements we get from a special light sectioning. Because the light sectioning only provides with weak $3 \mathrm{~d}$ measurements we introduced a simplified surface model of bounded books and developed a new dewarping method based on a weighted linear approximation along the vertical book length. Thereby we additionally described a technique to detect the geometrical location of the book crease. For the common application of book scanning our method yields well dewarped and aligned output results. There are only very few cases for which our method is not suitable.

If the technique will be implemented in a book scanner product we hope for extensive feed back from the users regarding the quality of our method. Furthermore we will do some impartial investigations about the OCR recognition. Depending on the geometric form we presume a significant improvement of the OCR recognition near the book crease.

To further the improvement of our dewarping system we have to upgrade the quality of the $3 \mathrm{~d}$ measurement. One way is to support the light sectioning by an additional area correlation method to reduce the weak measurements. The scanner hardware already fulfilled all conditions for this approach. Another idea is to use a second matrix camera. This brings less weak measurements because we can use pure stereo photogrammetry instead of light sectioning. But it increases the cost of the book scanner system. And it was a major concern designing the book scanner with a very good cost-benefit ratio.

\section{Acknowledgment}

This work was supported by AiF/Germany grants (FKZ: KF0056101SS4). We thank the company Chromasens for providing us with the book scanner for research purposes. And special thanks to Roger Klein.

\section{References}

1. Wada, T., Ukida, H., Matsuyama, T.: Shape from shading with interreflections under a proximal light source: Distorsion-free copying of an unfolded book. International Journal of Computer Vision 24(2), 125-135 (1997) 
2. Tan, C.L., Zhang, L., Zhang, Z., Xia, T.: Restoring warped document images through $3 \mathrm{~d}$ shape modeling. IEEE Transactions on Pattern Analysis and Machine Intelligence 28(2), 195-208 (2006)

3. Wu, C., Agam, G.: Document image de-warping for text/graphics recognition. In: Proc. of Joint IAPR and SPR, pp. 348-357 (2002)

4. Zhang, Z., Tan, C.L.: Correcting document image warping based on regression of curved text lines. In: Proc. of 7th International Conference on Document Analysis and Recognition, vol. 1, pp. 589-595 (2003)

5. Ulges, A., Lampert, C.H., Breuel, T.M.: Document image dewarping using robust estimation of curled text lines. In: Proc. of 8th International Conference on Document Analysis and Recognition, vol. 2, pp. 1001-1005. IEEE Computer Society, Washington, DC (2005)

6. Rebmann, R., Michaelis, B., Krell, G., Seiffert, U., Püschel, F.: Improving image processing systems by artificial neural networks. In: Dengel, A., Junker, M., Weisbecker, A. (eds.) Reading and Learning. LNCS, vol. 2956, pp. 37-64. Springer, Heidelberg (2004)

7. Frei, B.: Method and device for the correction of a scanned image. Patent US020050053304A1 (2002)

8. Donescu, A., Bouju, A., Quillet, V.: Former books digital processing: image warping. In: Proc. Workshop on Document Image Analysis, pp. 5-9 (1997)

9. Yamashita, A., Kawarago, A., Kaneko, T., Miura, K.: Shape reconstruction and image restoration for non-flat surfaces of documents with a stereo vision system. In: Proc. of 17th International Conference on Pattern Recognition, vol. 1, pp. 486-489. IEEE Computer Society, Washington, DC (2004)

10. Chu, K.B., Zhang, L., Zhang, Y., Tan, C.L.: A fast and stable approach for restoration of warped document images. In: Proceedings of the Eighth International Conference on Document Analysis and Recognition, pp. 384-388. IEEE Computer Society, Washington, DC (2005)

11. Klette, R., Koschan, A., Schlüns, K.: Copmuter Vision: Three-Dimensional Data from Images. Springer, Heidelberg (1998)

12. Brown, M., Seales, W.: Image restoration of arbitrarily warped documents. IEEE Transactions on Pattern Analysis and Machine Intelligence 26(10), 1295-1306 (2004)

13. Liang, J., DeMenthon, D., Doermann, D.: Flattening curved documents in images. In: Proceedings of the 2005 IEEE Computer Society Conference on Computer Vision and Pattern Recognition, vol. 2, pp. 338-345 (2005)

14. Liang, J., DeMenthon, D., Doermann, D.: Geometric rectification of cameracaptured document images. IEEE Transactions on Pattern Analysis and Machine Intelligence (Preprint, 2007)

15. Lilienblum, E., Michaelis, B.: Digitalisation of warped documents supported by 3dsurface reconstruction. In: The 5th International Conference on Computer Vision Systems Conference Paper (2007), doi:10.2390/biecoll-icvs2007-69

16. Lilienblum, E., Michaelis, B., Schnitzlein, M.: Verfahren zum Entzerren einer mittels einer Scanneinrichtung eingescannten zweidimensionalen Abbildung einer nicht ebenflächigen Vorlage. German patent, No. 102006032533 (2006)

17. Shirai, Y.: Recognition of polyhedrons with a range finder. Pattern Recognition 4, 243-250 (1972) 